ESAIM: COCV 18 (2012) 954-968

DOI: $10.1051 / \mathrm{cocv} / 2011203$
ESAIM: Control, Optimisation and Calculus of Variations

www.esaim-cocv.org

\title{
CONTINUOUS DEPENDENCE ESTIMATES FOR THE ERGODIC PROBLEM OF BELLMAN-ISAACS OPERATORS VIA THE PARABOLIC CAUCHY PROBLEM*
}

\author{
Claudio Marchi ${ }^{1}$
}

\begin{abstract}
This paper concerns continuous dependence estimates for Hamilton-Jacobi-Bellman-Isaacs operators. We establish such an estimate for the parabolic Cauchy problem in the whole space $[0,+\infty) \times$ $\mathbb{R}^{n}$ and, under some periodicity and either ellipticity or controllability assumptions, we deduce a similar estimate for the ergodic constant associated to the operator. An interesting byproduct of the latter result will be the local uniform convergence for some classes of singular perturbation problems.
\end{abstract}

Mathematics Subject Classification. 35B25, 35B30, 35J60, 35K55, 49L25, 49N70.

Received December 23, 2010. Revised November 14, 2011.

Published online 16 January 2012.

\section{INTRODUCTION}

This paper is devoted to continuous dependence estimates for two problems for Hamilton-Jacobi-BellmanIsaacs (briefly, HJBI) operators: the parabolic Cauchy problem for any time and, mainly, the associated ergodic constant. For the former issue, we consider the Cauchy problem

$$
\begin{cases}\partial_{t} u+H\left(x, D u, D^{2} u\right)=0 & \text { in }(0,+\infty) \times \mathbb{R}^{n} \\ u(0, x)=0 & \text { on } \mathbb{R}^{n}\end{cases}
$$

for the HJBI operator

$$
H(x, p, X)=\min _{\beta \in B} \max _{\alpha \in A}\{-\operatorname{tr}(a(x, \alpha, \beta) X)+f(x, \alpha, \beta) \cdot p+\ell(x, \alpha, \beta)\}
$$

where $\partial_{t} \equiv \partial / \partial t, D u$ and $D^{2} u$ stand respectively for the gradient and for the Hessian matrix of the real-valued function $u=u(t, x)$.

For instance, problems of this kind naturally arise in zero-sum two-persons stochastic differential games: consider the control system for $s>0$

$$
\mathrm{d} x_{s}=f\left(x_{s}, \alpha_{s}, \beta_{s}\right) \mathrm{d} s+\sqrt{2} \sigma\left(x_{s}, \alpha_{s}, \beta_{s}\right) \mathrm{d} W_{s}, \quad x_{0}=x
$$

Keywords and phrases. Continuous dependence estimates, parabolic Hamilton-Jacobi equations, viscosity solutions, ergodic problems, differential games, singular perturbations.

* Work partially supported by the INDAM-GNAMPA project "Fenomeni di propagazione di fronti e problemi di omogeneizzazione" and by the Fondazione CaRiPaRo Project "Nonlinear Partial Differential Equations: models, analysis, and controltheoretic problems".

1 Dip. di Matematica Pura ed Applicata, Università di Padova, via Trieste 63, 35121 Padova, Italy. marchi@math.unipd.it 
where $(\Omega, \mathcal{F}, \mathcal{P})$ is a probability space endowed with a right continuous filtration $\left(\mathcal{F}_{t}\right)_{0 \leq t<+\infty}$ and a $p$ dimensional Brownian motion $W_{t}$. The control law $\alpha$ (respectively, $\beta$ ) belongs to the set $\mathcal{A}$ (resp., $\mathcal{B}$ ) of progressively measurable processes which take value in the compact set $A$ (resp., $B$ ). The controls $\alpha$ and $\beta$ are chosen respectively by the first and the second player whose purposes are opposite: the former wants to minimize the cost functional

$$
P(t, x, \alpha, \beta):=\mathbb{E}_{x} \int_{0}^{t} \ell\left(x_{s}, \alpha_{s}, \beta_{s}\right) \mathrm{d} s
$$

(here, $\mathbb{E}_{x}$ denotes the expectation) while the latter wants to maximize it. It is well known (see [22]) that the lower value function

$$
u(t, x):=\inf _{\alpha \in \Gamma} \sup _{\beta \in \mathcal{B}} P(t, x, \alpha[\beta], \beta)
$$

is a viscosity solution to problem (1.1)-(1.2) with $a=\sigma \sigma^{T}$ where $\Gamma$ stands for the set of admissible strategies of the first player (namely, nonanticipating maps $\alpha: \mathcal{B} \rightarrow \mathcal{A}$; see [22]).

For the latter issue (namely, the ergodic problem for $H$ ), we seek a pair $(v, U)$, with $v \in C\left(\mathbb{R}^{n}\right)$ and $U \in \mathbb{R}$ (called the ergodic constant) which, in viscosity sense, satisfies

$$
H\left(x, D v, D^{2} v\right)=U \quad \text { in } \mathbb{R}^{n} .
$$

Let us recall $([2,3])$ that, in terms of stochastic differential games, the constant $U$ is characterized by the following limits (uniform in $x$ )

$$
U=\lim _{t \rightarrow+\infty} \inf _{\alpha \in \Gamma} \sup _{\beta \in \mathcal{B}} t^{-1} \mathbb{E}_{x} \int_{0}^{t} \ell\left(x_{s}, \alpha[\beta]_{s}, \beta_{s}\right) \mathrm{d} s=\lim _{\delta \rightarrow 0^{+}} \inf _{\alpha \in \Gamma} \sup _{\beta \in \mathcal{B}} \mathbb{E}_{x} \delta \int_{0}^{+\infty} \ell\left(x_{s}, \alpha[\beta]_{s}, \beta_{s}\right) \mathrm{e}^{-\delta s} \mathrm{~d} s .
$$

In other words, by standard theory (see [22]), $U$ fulfills

$$
U=\lim _{t \rightarrow+\infty} u(t, x) / t=\lim _{\delta \rightarrow 0^{+}} \delta w_{\delta}(x) \quad \text { uniformly in } x
$$

where $u$ and $w_{\delta}$ are respectively the solution to problem (1.1) and to equation

$$
\delta w_{\delta}+H\left(x, D w_{\delta}, D^{2} w_{\delta}\right)=0 \quad \text { in } \mathbb{R}^{n} .
$$

The continuous dependence estimates for fully nonlinear equations have been widely studied in literature, starting from the paper by Souganidis [34] for first-order equations. In fact, such estimates play a crucial role in many contexts as error estimates for approximation schemes (see [7,19] and references therein), regularity results (for instance, see $[8,13,27])$ and rate of convergence for vanishing viscosity methods (see $[15,23,27,28]$ and references therein). In particular, let us recall that Cockburn et al. [15] tackled up the continuous dependence estimate for quasi-linear second-order equations with Neumann boundary conditions, while Grinpenberg [23] addressed the case of the Dirichlet boundary data for the same equations. Afterwards, Jakobsen and Karlsen [27] extended their results to more general classes of equations (see also [28] for elliptic problems; we refer the reader to the papers $[12,29]$ for integro-differential HJB equations). Furthermore, Jakobsen and Georgelin [26] extended the previous results to problems with more general boundary conditions and domains.

On the other hand, the ergodic problem has been widely studied in connection with homogenization or singular perturbation problems (see $[1,2,6,11,14,21,30,32]$ and references therein), with long-time behavior of solutions to parabolic equations (for instance, see $[3,5,9]$ ) and with dynamical systems in a torus (see $[4,16,33]$ ). It is well known (see $[2,3]$ ) that, under some periodicity and either ellipticity or controllability assumptions, there exists exactly one value $U \in \mathbb{R}$ such that equation (1.5) admits at least one bounded solution. For the elliptic case, Evans [21] obtained a continuous dependence estimate for the ergodic constant for operators which are Lipschitz continuous in the variable $x$ uniformly with respect to $(p, X)$. Afterwards, Alvarez and Bardi [2], Theorem 4.5, extended his result to operators which are elliptic, convex in $X$ and with a Hölder continuous 
running cost $\ell$ (see also [2], Cor. 4.3 and Thm. 2.10, for other generalizations in different directions). For the controllable case, Alvarez and Bardi [2], Corollary 6.9, established a continuous dependence estimate for the ergodic constant provided that the dispersion matrix $\sigma$ is left unchanged. Let us observe that, in all these papers, the regularity of the ergodic constant is ensured by a straightforward application of the comparison principle.

This paper is devoted to two purposes. The former is to establish a continuous dependence estimate for problem (1.1)-(1.2) (namely, an estimate of $\|u-v\|_{\infty}$, where $u$ and $v$ are solutions to two equations (1.1) with different coefficients) with the following three features: the dependence on the $\mathbb{L}^{\infty}$-distance between the coefficients is explicit, the constants can be explicitly characterized and, above all, the estimate holds in the whole space $[0,+\infty) \times \mathbb{R}^{n}$ and grows at most linearly in $t$. As far as we known, these features (especially the last one) have never been tackled up in literature.

The latter purpose (which we consider the main one) is to establish a continuous dependence estimate for the ergodic constant associated to HJBI operators as in (1.2) only under periodicity and ellipticity/controllability assumptions. An interesting byproduct will be the local uniform convergence for some classes of singular perturbation problems (see Sect. 3.1 for the precise definition). The most important contributions on this matter are: our approach is completely different from the ones in literature and we deal with HJBI operators in full generality (both for the dependence of the ergodic constant and for the convergence of singular perturbation problems).

This paper is organized as follows: in the rest of this section, we provide some notations and list the standing assumptions. Section 2 contains the continuous dependence estimate for the parabolic Cauchy problem (1.1) and its application to some degenerate problems as well. Section 3 concerns the continuous dependence estimate for the ergodic constant in (1.5); Section 3.1 is devoted to illustrate how to derive the local uniform convergence for singular perturbation problems.

\subsection{Notations and standing assumptions}

Notations: We define $\mathbb{M}^{n, p}$ and $\mathbb{S}^{n}$ respectively as the set of $n \times p$ real matrices and the set of $n \times n$ symmetric matrices. The latter is endowed with the Euclidean norm and with the usual order, namely: for $X=\left(X_{i j}\right)_{i, j=1, \ldots, n} \in \mathbb{S}^{n},\|X\|:=\left(\sum_{i, j=1}^{n} X_{i j}^{2}\right)^{1 / 2} \equiv \operatorname{tr}\left(X X^{T}\right)^{1 / 2}$ and, for $X, Y \in \mathbb{S}^{n}$, we shall write $X \geq Y$, if $X-Y$ is a semi-definite positive matrix. We denote by $I_{n}$ the identity matrix in $\mathbb{S}^{n}$; we shall simply use $I$ when there is no ambiguity.

For every positive $t$, we set $Q_{t}:=[0, t) \times \mathbb{R}^{n}$ and $Q_{\infty}:=[0,+\infty) \times \mathbb{R}^{n}$.

For every real-valued function $h$, we set $\|h\|_{\infty}:=\operatorname{ess} \sup |h(y)|$; for $\gamma \in(0,1]$, we use the $\gamma$-Hölder norm: $|h|_{\gamma}:=\sup _{y \neq x} \frac{|h(y)-h(x)|}{|y-x|^{\gamma}}$. Moreover, $J^{2,+} h(\xi)$ and $J^{2,-} h(\xi)$ stand respectively for the second-order superjet and subjet of $h$ at the point $\xi$ (see [17], Sect. 2, for the precise definition and main properties). For each function $h$ defined on $Q_{t}, \mathcal{P}^{2,+} h(\tau, \xi)$ and $\mathcal{P}^{2,-} h(\tau, \xi)$ denote respectively the parabolic super- and subjects at the point $(\tau, \xi)$ (see [17], Sect. 8). A real function $\omega$ is said a modulus of continuity whenever it is a nonnegative continuous non-decreasing function on $[0,+\infty)$ with $\omega(0)=0$.

Standing assumptions: for the operator $H$ in (1.2), the following assumptions will hold throughout this paper

(A1) $A$ and $B$ are two compact metric spaces.

(A2) $a=\sigma \sigma^{T}$. The functions $\sigma, f$ and $\ell$ are bounded continuous functions in $\mathbb{R}^{n} \times A \times B$ with value respectively in $\mathbb{M}^{n, p}, \mathbb{R}^{n}$, and $\mathbb{R}$; namely, for some $C>0$, there holds: $\|\sigma\|_{\infty},\|f\|_{\infty},\|\ell\|_{\infty} \leq C$.

(A3) The drift vectors $f$ and the dispersion matrix $\sigma$ are Lipschitz continuous in $x$ uniformly in $(\alpha, \beta)$, namely: for some positive constant $C_{\phi}$, every $\phi=\sigma, f$ satisfies

$$
|\phi(x, \alpha, \beta)-\phi(y, \alpha, \beta)| \leq C_{\phi}|x-y| \quad \forall x, y \in \mathbb{R}^{n}, \forall(\alpha, \beta) \in A \times B .
$$

The running cost $\ell$ is uniformly continuous in $x$ uniformly in $(\alpha, \beta)$, namely: there exists a modulus of continuity $\omega$ such that

$$
|\ell(x, \alpha, \beta)-\ell(y, \alpha, \beta)| \leq \omega(|x-y|) \quad \forall x, y \in \mathbb{R}^{n}, \forall(\alpha, \beta) \in A \times B .
$$




\section{Estimate for the parabolic Cauchy problem}

For $i=1,2$, consider the parabolic Cauchy problems

$$
\begin{cases}\partial_{t} u^{i}+\min _{\beta \in B} \max _{\alpha \in A}\left\{-\operatorname{tr}\left(a_{i}(x, \alpha, \beta) D^{2} u^{i}\right)+f_{i}(x, \alpha, \beta) \cdot D u^{i}+\ell_{i}(x, \alpha, \beta)\right\}=0 & \text { in } Q_{\infty} \\ u^{i}(0, x)=0 & \text { on } \mathbb{R}^{n}\end{cases}
$$

where the coefficients fulfill our standing assumptions $(A 1)-(A 3)$. The main purpose of this section is to provide an estimate of $\left\|u^{1}(t, \cdot)-u^{2}(t, \cdot)\right\|_{\infty}$ for every $t \in[0,+\infty)$. Such an estimate could be obtained as a straightforward application of the comparison principle provided that some bound on the $C^{2}$-norm of the solution is available. Unfortunately, this is not the case for operators (1.2). In fact, our approach is based on the comparison principle techniques for viscosity solutions (see [17]): doubling the variables and adding a penalization term. Let us observe that this approach does not require the non-degeneracy of the operator $H$; actually, in Section 2.1, we shall also apply our result to some degenerate problems.

Remark 2.1. By standard viscosity theory (for instance, see [17]), assumptions $(A 1)-(A 3)$ guarantee that the comparison principle applies to problem $(\mathrm{Pi})$; whence, by the Perron method, one can easily deduce that $(\mathrm{Pi})$ admits exactly one solution $u^{i} \in C\left(Q_{\infty}\right)$ with

$$
\left|u^{i}(t, x)\right| \leq t C, \quad \forall(t, x) \in Q_{\infty}
$$

where $C$ is the constant introduced in assumption $(A 2)$.

Theorem 2.2. Let $u^{i}$ be the unique solution to problem $(\mathrm{Pi})$ which satisfies the bound $(2.1)(i=1,2)$. Furthermore, let us assume that, for some $\gamma \in(0,1], u^{i}(t, \cdot)$ is $\gamma$-Hölder continuous uniformly in $t$, namely: for some $C_{H}>0$, there holds

$$
\left|u^{i}(t, \cdot)\right|_{\gamma} \leq C_{H}, \quad \forall t \in[0,+\infty), i=1,2 .
$$

Then, there exist a positive constant $M$ such that, for every $(x, t) \in Q_{\infty}$, there holds

$$
\begin{aligned}
\left|u^{1}(t, x)-u^{2}(t, x)\right| \leq & t M\left[\max _{x, \alpha, \beta}\left\|\sigma_{1}-\sigma_{2}\right\|^{\gamma}+\max _{x, \alpha, \beta}\left|f_{1}-f_{2}\right|^{\gamma}+\max _{x, \alpha, \beta}\left|\ell_{1}-\ell_{2}\right|\right. \\
& \left.+\omega\left(\max _{x, \alpha, \beta}\left\|\sigma_{1}-\sigma_{2}\right\|+\max _{x, \alpha, \beta}\left|f_{1}-f_{2}\right|\right)\right] .
\end{aligned}
$$

Proof of Theorem 2.2. We shall argue using some techniques introduced in [15,27]. Fix $t>0$ and set $E_{t}:=$ $[0, t) \times \mathbb{R}^{n} \times \mathbb{R}^{n}$. For every $\eta \in(0,+\infty)$ and $\varepsilon \in(0,1)$, we introduce

$$
s_{t}:=\sup _{E_{t}}\left\{u^{1}(\tau, x)-u^{2}(\tau, y)-\left(\frac{\eta}{2}|x-y|^{2}+\frac{\varepsilon}{2}\left(|x|^{2}+|y|^{2}\right)+\frac{\varepsilon}{t-\tau}\right)\right\} .
$$

Our first purpose is to establish an upper bound for $s_{t}$. To this end, without any loss of generality, we can assume $s_{t}>0$. For $\delta \in(0,1)$, define

$$
\psi(\tau, x, y):=u^{1}(\tau, x)-u^{2}(\tau, y)-\frac{\delta s_{t} \tau}{t}-\left(\frac{\eta}{2}|x-y|^{2}+\frac{\varepsilon}{2}\left(|x|^{2}+|y|^{2}\right)+\frac{\varepsilon}{t-\tau}\right) .
$$

Let us observe that definition (2.3) entails

$$
\begin{aligned}
\sup _{E_{t}} \psi \geq & \sup _{E_{t}}\left\{u^{1}(\tau, x)-u^{2}(\tau, y)-\left(\frac{\eta}{2}|x-y|^{2}+\frac{\varepsilon}{2}\left(|x|^{2}+|y|^{2}\right)+\frac{\varepsilon}{t-\tau}\right)\right\} \\
& -\sup _{\tau \in[0, t)}\left\{\frac{\delta s_{t} \tau}{t}\right\} \\
& \geq(1-\delta) s_{t}>0 .
\end{aligned}
$$


Since the functions $u^{1}$ and $u^{2}$ are bounded in $Q_{t}$ and since the function $\psi$ tends to $-\infty$ both as $\tau \rightarrow t^{-}$and as $|x|+|y| \rightarrow+\infty$, we deduce that there exists a point $\left(\tau_{0}, x_{0}, y_{0}\right) \in E_{t}$ where the function $\psi$ attains its global maximum, namely

$$
\psi\left(\tau_{0}, x_{0}, y_{0}\right)=\sup _{E_{t}} \psi \geq 0
$$

where the inequality is due to relation $(2.5)$.

Let us now claim that, for $\bar{C}:=\left(2 C_{H}\right)^{1 /(2-\gamma)}$, there holds

$$
\left|x_{0}-y_{0}\right| \leq \bar{C} \eta^{-1 /(2-\gamma)}, \quad \varepsilon\left(\left|x_{0}\right|^{2}+\left|y_{0}\right|^{2}\right) \leq 4 C t
$$

where $C_{H}, \gamma$ and $C$ are the constants introduced respectively in assumption (2.2) and (A2); in particular, let us emphasize that $\bar{C}$ is independent of $t$. Actually, in order to prove the former estimate, we observe that inequality $\psi\left(\tau_{0}, x_{0}, x_{0}\right)+\psi\left(\tau_{0}, y_{0}, y_{0}\right) \leq 2 \psi\left(\tau_{0}, x_{0}, y_{0}\right)$ and assumption $(2.2)$ give

$$
\begin{aligned}
\eta\left|x_{0}-y_{0}\right|^{2} & \leq\left[u^{1}\left(\tau_{0}, x_{0}\right)-u^{1}\left(\tau_{0}, y_{0}\right)\right]+\left[u^{2}\left(\tau_{0}, x_{0}\right)-u^{2}\left(\tau_{0}, y_{0}\right)\right] \\
& \leq 2 C_{H}\left|x_{0}-y_{0}\right|^{\gamma}
\end{aligned}
$$

Let us now prove the latter estimate in (2.7): by estimates (2.1) and (2.6), we infer

$$
\varepsilon\left(\left|x_{0}\right|^{2}+\left|y_{0}\right|^{2}\right) \leq 2 u^{1}\left(\tau_{0}, x_{0}\right)-2 u^{2}\left(\tau_{0}, y_{0}\right) \leq 4 C t .
$$

Hence, the proof of estimates (2.7) is accomplished.

We introduce the test function

$$
\phi(\tau, x, y):=\frac{\delta s_{t} \tau}{t}+\frac{\eta}{2}|x-y|^{2}+\frac{\varepsilon}{2}\left(|x|^{2}+|y|^{2}\right)+\frac{\varepsilon}{t-\tau}
$$

and we invoke [17], Theorem 8.3: for every $\nu>0$, there exist values $a, b \in \mathbb{R}$ and matrices $X, Y \in \mathbb{S}^{n}$ such that

$$
\begin{gathered}
\left(a, D_{x} \phi\left(\tau_{0}, x_{0}, y_{0}\right), X\right) \in \mathcal{P}^{2,+} u^{1}\left(\tau_{0}, x_{0}\right), \quad\left(b, D_{y} \phi\left(\tau_{0}, x_{0}, y_{0}\right), Y\right) \in \mathcal{P}^{2,-} u^{2}\left(\tau_{0}, y_{0}\right), \\
a-b=\partial_{\tau} \phi\left(\tau_{0}, x_{0}, y_{0}\right) \equiv \frac{\delta s_{t}}{t}+\frac{\varepsilon}{\left(t-\tau_{0}\right)^{2}} \\
\left(\begin{array}{cc}
X & 0 \\
0 & -Y
\end{array}\right) \leq \Theta+\nu \Theta^{2}
\end{gathered}
$$

where $\Theta:=\eta\left(\begin{array}{cc}I & -I \\ -I & I\end{array}\right)+\varepsilon\left(\begin{array}{ll}I & 0 \\ 0 & I\end{array}\right)$. From the last inequality, one can deduce that, for every $(\alpha, \beta) \in A \times B$, there holds

$$
\operatorname{tr}\left(a_{1}\left(x_{0}, \alpha, \beta\right) X\right)-\operatorname{tr}\left(a_{2}\left(y_{0}, \alpha, \beta\right) Y\right) \leq \eta\left\|\sigma_{1}\left(x_{0}, \alpha, \beta\right)-\sigma_{2}\left(y_{0}, \alpha, \beta\right)\right\|^{2}+2 \varepsilon C^{2}+\nu \operatorname{tr}\left(\Sigma \Theta^{2}\right)
$$

with

$$
\Sigma:=\left(\begin{array}{cc}
\sigma_{1}\left(x_{0}, \alpha, \beta\right) \sigma_{1}\left(x_{0}, \alpha, \beta\right)^{T} & \sigma_{2}\left(y_{0}, \alpha, \beta\right) \sigma_{1}\left(x_{0}, \alpha, \beta\right)^{T} \\
\sigma_{1}\left(x_{0}, \alpha, \beta\right) \sigma_{2}\left(y_{0}, \alpha, \beta\right)^{T} & \sigma_{2}\left(y_{0}, \alpha, \beta\right) \sigma_{2}\left(y_{0}, \alpha, \beta\right)^{T}
\end{array}\right) .
$$

In order to prove this inequality, we shall use the arguments by Ishii [24]. Multiplying relation (2.11) by matrix $\Sigma$ (which is symmetric and nonnegative definite) and evaluating the trace, we obtain

$$
\begin{aligned}
& \operatorname{tr}\left(\sigma_{1}\left(x_{0}, \alpha, \beta\right) \sigma_{1}\left(x_{0}, \alpha, \beta\right)^{T} X-\sigma_{2}\left(y_{0}, \alpha, \beta\right) \sigma_{2}\left(y_{0}, \alpha, \beta\right)^{T} Y\right) \\
& \leq \eta \operatorname{tr}[ {\left[\left(\sigma_{1}\left(x_{0}, \alpha, \beta\right)-\sigma_{2}\left(y_{0}, \alpha, \beta\right)\right)\left(\sigma_{1}\left(x_{0}, \alpha, \beta\right)-\sigma_{2}\left(y_{0}, \alpha, \beta\right)\right)^{T}\right] } \\
&+\varepsilon \operatorname{tr}\left(\sigma_{1}\left(x_{0}, \alpha, \beta\right) \sigma_{1}\left(x_{0}, \alpha, \beta\right)^{T}\right)+\varepsilon \operatorname{tr}\left(\sigma_{2}\left(y_{0}, \alpha, \beta\right) \sigma_{2}\left(y_{0}, \alpha, \beta\right)^{T}\right)+\nu \operatorname{tr}\left(\Sigma \Theta^{2}\right)
\end{aligned}
$$


therefore, by assumption $(A 2)$, relation (2.12) easily follows.

Since $u^{1}$ is a subsolution to problem (Pi) with $i=1$, the former relation in (2.9) yields

$$
\begin{aligned}
0 \geq & a+\min _{\beta \in B} \max _{\alpha \in A}\left\{-\operatorname{tr}\left(a_{1}\left(x_{0}, \alpha, \beta\right) X\right)+f_{1}\left(x_{0}, \alpha, \beta\right) \cdot D_{x} \phi\left(\tau_{0}, x_{0}, y_{0}\right)+\ell_{1}\left(x_{0}, \alpha, \beta\right)\right\} \\
\geq & b+\min _{\beta \in B} \max _{\alpha \in A}\left\{-\operatorname{tr}\left(a_{2}\left(y_{0}, \alpha, \beta\right) Y\right)+f_{1}\left(x_{0}, \alpha, \beta\right) \cdot\left(\eta\left(x_{0}-y_{0}\right)+\varepsilon x_{0}\right)+\ell_{1}\left(x_{0}, \alpha, \beta\right)\right\} \\
& -\eta \max _{\alpha, \beta}\left\|\sigma_{1}\left(x_{0}, \alpha, \beta\right)-\sigma_{2}\left(y_{0}, \alpha, \beta\right)\right\|^{2}-2 \varepsilon C^{2}-\nu \max _{\alpha, \beta} \operatorname{tr}\left(\Sigma \Theta^{2}\right)+\frac{\delta s_{t}}{t}+\frac{\varepsilon}{\left(t-\tau_{0}\right)^{2}}
\end{aligned}
$$

where the last inequality is due to the definition of $\phi(2.8)$ and to relations (2.10) and (2.12). Since $u^{2}$ is a supersolution to equation ( $\mathrm{Pi}$ ) with $i=2$, by assumption $(A 2)$, last inequality entails the following upper bound for $s_{t}$ :

$$
\begin{aligned}
\frac{\delta s_{t}}{t}+\frac{\varepsilon}{\left(t-\tau_{0}\right)^{2}} \leq & \eta \max _{\alpha, \beta}\left\|\sigma_{1}\left(x_{0}, \alpha, \beta\right)-\sigma_{2}\left(y_{0}, \alpha, \beta\right)\right\|^{2}+2 \varepsilon C^{2}+\nu \max _{\alpha, \beta} \operatorname{tr}\left(\Sigma \Theta^{2}\right) \\
& +\varepsilon C\left(\left|x_{0}\right|+\left|y_{0}\right|\right)+\eta\left|x_{0}-y_{0}\right| \max _{\alpha, \beta}\left|f_{1}\left(x_{0}, \alpha, \beta\right)-f_{2}\left(y_{0}, \alpha, \beta\right)\right| \\
& +\max _{\alpha, \beta}\left|\ell_{1}\left(x_{0}, \alpha, \beta\right)-\ell_{2}\left(y_{0}, \alpha, \beta\right)\right| .
\end{aligned}
$$

Owing to the definition of $s_{t}$ in $(2.3)$, we have

$$
u^{1}(\tau, x)-u^{2}(\tau, y)-\frac{\eta}{2}|x-y|^{2}-\frac{\varepsilon}{2}\left(|x|^{2}+|y|^{2}\right) \leq s_{t}+\frac{\varepsilon}{t-\tau} \quad \forall(\tau, x, y) \in E_{t} .
$$

Taking into account the last two inequalities, for every $(\tau, x) \in Q_{t}$, we infer

$$
\begin{aligned}
u^{1}(\tau, x)-u^{2}(\tau, x) \leq & \frac{t}{\delta}\left[\eta \max _{\alpha, \beta}\left\|\sigma_{1}\left(x_{0}, \alpha, \beta\right)-\sigma_{2}\left(y_{0}, \alpha, \beta\right)\right\|^{2}+2 \varepsilon C^{2}+\nu \max _{\alpha, \beta} \operatorname{tr}\left(\Sigma \Theta^{2}\right)\right. \\
& +\eta\left|x_{0}-y_{0}\right| \max _{\alpha, \beta}\left|f_{1}\left(x_{0}, \alpha, \beta\right)-f_{2}\left(y_{0}, \alpha, \beta\right)\right|+\varepsilon C\left(\left|x_{0}\right|+\left|y_{0}\right|\right) \\
& \left.+\max _{\alpha, \beta}\left|\ell_{1}\left(x_{0}, \alpha, \beta\right)-\ell_{2}\left(y_{0}, \alpha, \beta\right)\right|\right]+\frac{\varepsilon}{t-\tau}+\varepsilon|x|^{2} .
\end{aligned}
$$

By the regularity of the coefficients (see assumption $(A 3)$ ) and estimate (2.7), for $\tilde{C}:=2 C_{\sigma}^{2} \bar{C}^{2}+2+C_{f} \bar{C}^{2}+\bar{C}$, we get

$$
\begin{aligned}
u^{1}(\tau, x)-u^{2}(\tau, x) \leq & \frac{t}{\delta}\left[2 \eta\left(C_{\sigma}^{2}\left|x_{0}-y_{0}\right|^{2}+\max _{x, \alpha, \beta}\left\|\sigma_{1}-\sigma_{2}\right\|^{2}\right)+\eta C_{f}\left|x_{0}-y_{0}\right|^{2}+\eta\left|x_{0}-y_{0}\right| \max _{x, \alpha, \beta}\left|f_{1}-f_{2}\right|\right. \\
& \left.+\omega\left(\left|x_{0}-y_{0}\right|\right)+\max _{x, \alpha, \beta}\left|\ell_{1}-\ell_{2}\right|\right]+\varepsilon\left[\frac{1}{t-\tau}+\frac{C t}{\delta}\left(\left|x_{0}\right|+\left|y_{0}\right|+2 C\right)+|x|^{2}\right] \\
& +\nu t \delta^{-1} \max _{\alpha, \beta} \operatorname{tr}\left(\Sigma \Theta^{2}\right) \\
\leq & \frac{t}{\delta} \tilde{C}\left[\eta^{-\gamma /(2-\gamma)}+\eta \max _{x, \alpha, \beta}\left\|\sigma_{1}-\sigma_{2}\right\|^{2}+\eta^{(1-\gamma) /(2-\gamma)} \max _{x, \alpha, \beta}\left|f_{1}-f_{2}\right|\right] \\
& +\frac{t}{\delta}\left[\max _{x, \alpha, \beta}\left|\ell_{1}-\ell_{2}\right|+\omega\left(\bar{C} \eta^{-1 /(2-\gamma)}\right)\right]+\varepsilon\left[\frac{1}{t-\tau}+\frac{C t}{\delta}\left(\left|x_{0}\right|+\left|y_{0}\right|+2 C\right)+|x|^{2}\right] \\
& +\nu t \delta^{-1} \max _{\alpha, \beta} \operatorname{tr}\left(\Sigma \Theta^{2}\right) .
\end{aligned}
$$


Letting $\nu \rightarrow 0^{+}$and afterwards $\varepsilon \rightarrow 0^{+}$, by estimate (2.7), we infer

$$
\begin{aligned}
u^{1}(\tau, x)-u^{2}(\tau, x) \leq & \frac{t}{\delta} \tilde{C}\left[\eta^{-\gamma /(2-\gamma)}+\eta \max _{x, \alpha, \beta}\left\|\sigma_{1}-\sigma_{2}\right\|^{2}+\eta^{(1-\gamma) /(2-\gamma)} \max _{x, \alpha, \beta}\left|f_{1}-f_{2}\right|\right] \\
& +\frac{t}{\delta}\left[\max _{x, \alpha, \beta}\left|\ell_{1}-\ell_{2}\right|+\omega\left(\bar{C} \eta^{-1 /(2-\gamma)}\right)\right] .
\end{aligned}
$$

Letting $\delta \rightarrow 1^{-}$and afterwards $\tau \rightarrow t^{-}$, by the continuity of the functions $u^{1}$ and $u^{2}$, for every $x \in \mathbb{R}^{n}$, we deduce

$$
\begin{aligned}
u^{1}(t, x)-u^{2}(t, x) \leq & t \tilde{C}\left[\eta^{-\gamma /(2-\gamma)}+\eta \max _{x, \alpha, \beta}\left\|\sigma_{1}-\sigma_{2}\right\|^{2}+\eta^{(1-\gamma) /(2-\gamma)} \max _{x, \alpha, \beta}\left|f_{1}-f_{2}\right|\right] \\
& +t\left[\max _{x, \alpha, \beta}\left|\ell_{1}-\ell_{2}\right|+\omega\left(\bar{C} \eta^{-1 /(2-\gamma)}\right)\right] .
\end{aligned}
$$

We shall consider separately four cases; $\overline{\bar{C}}$ will be a constant depending only on $\bar{C}$ and $\gamma$. (i) For $\gamma=1$ and $\sigma_{1}=\sigma_{2}$, as $\eta \rightarrow+\infty$, relation (2.13) becomes

$$
u^{1}(t, x)-u^{2}(t, x) \leq t \tilde{C} \max _{x, \alpha, \beta}\left|f_{1}-f_{2}\right|+t \max _{x, \alpha, \beta}\left|\ell_{1}-\ell_{2}\right|
$$

(ii) for $\gamma=1$ and $\sigma_{1} \neq \sigma_{2}$, we choose $\eta=\bar{C} \max _{x, \alpha, \beta}\left\|\sigma_{1}-\sigma_{2}\right\|^{-1}$ and we get

$$
\begin{aligned}
u^{1}(t, x)-u^{2}(t, x) \leq & t \tilde{C} \overline{\bar{C}}\left[\max _{x, \alpha, \beta}\left\|\sigma_{1}-\sigma_{2}\right\|+\max _{x, \alpha, \beta}\left|f_{1}-f_{2}\right|\right] \\
& +t\left[\max _{x, \alpha, \beta}\left|\ell_{1}-\ell_{2}\right|+\omega\left(\max _{x, \alpha, \beta}\left\|\sigma_{1}-\sigma_{2}\right\|\right)\right] ;
\end{aligned}
$$

(iii) for $\gamma \in(0,1), \sigma_{1}=\sigma_{1}$ and $f_{1}=f_{2}$, as $\eta \rightarrow+\infty$, relation (2.13) becomes

$$
u^{1}(t, x)-u^{2}(t, x) \leq t \max _{x, \alpha, \beta}\left|\ell_{1}-\ell_{2}\right|
$$

(iv) for $\gamma \in(0,1)$ and $m:=\max \left\{\max _{x, \alpha, \beta}\left\|\sigma_{1}-\sigma_{2}\right\|, \max _{x, \alpha, \beta}\left|f_{1}-f_{2}\right|\right\}>0$, we choose $\eta=\left(\bar{C}^{-1} m\right)^{\gamma-2}$ and we obtain

$$
u^{1}(t, x)-u^{2}(t, x) \leq t \tilde{C} \overline{\bar{C}} m^{\gamma}+t\left[\max _{x, \alpha, \beta}\left|\ell_{1}-\ell_{2}\right|+\omega(m)\right] .
$$

Owing to the arbitrariness of the value $t$, one side of the inequality in our statement is completely proved. Being similar, the proof of the other one is omitted.

Remark 2.3. Actually, by the calculations of the proof above, for $\gamma=1$, we established

$$
\left|u^{1}(t, x)-u^{2}(t, x)\right| \leq t M\left[\max _{x, \alpha, \beta}\left\|\sigma_{1}-\sigma_{2}\right\|+\max _{x, \alpha, \beta}\left|f_{1}-f_{2}\right|\right]+t\left[\max _{x, \alpha, \beta}\left|\ell_{1}-\ell_{2}\right|+\omega\left(\max _{x, \alpha, \beta}\left\|\sigma_{1}-\sigma_{2}\right\|\right)\right]
$$

for every $(t, x) \in Q_{\infty}$.

Remark 2.4. In Theorem 2.2, the absence of zero-order term in (Pi) is crucial. Actually, if $u^{i}$ solves

$$
\partial_{t} u^{i}+\min _{\beta \in B} \max _{\alpha \in A}\left\{-\operatorname{tr}\left(a_{i} D^{2} u^{i}\right)+f_{i} \cdot D u^{i}+h_{i} u^{i}+\ell_{i}\right\}=0 \quad \text { in } Q_{\infty}, \quad u^{i}(0, x)=0 \quad \text { on } \mathbb{R}^{n}
$$

for some bounded function $h_{i}=h_{i}(x, \alpha, \beta)$, then the right-hand side in the statement of Theorem 2.2 may increase exponentially in $t$. Let us also observe that in this case, in general, estimate (2.1) fails and it should be replaced by: $\left|u^{i}(t, x)\right| \leq C e^{c t}$ for some constant $c$ depending on $\sup _{x, \alpha, \beta}\left|h_{i}(x, \alpha, \beta)\right|$. 


\subsection{Example: degenerate parabolic problems}

This Section is devoted to illustrate an application of Theorem 2.2 to some classes of parabolic Cauchy problems for degenerate HJBI operators.

Corollary 2.5. Besides our standing conditions, assume that, for some positive constants $\nu$ and $C_{1}$, there holds

$$
\min _{\beta \in B} \max _{\alpha \in A}\left\{-\operatorname{tr}\left(a_{i}(x, \alpha, \beta) X\right)+f_{i}(x, \alpha, \beta) \cdot p+\ell_{i}(x, \alpha, \beta)\right\} \geq \nu|p|-C_{1}
$$

for every $(x, p, X) \in \mathbb{R}^{n} \times \mathbb{R}^{n} \times \mathbb{S}^{n}(i=1,2)$. Then, there exists $M>0$ such that, for every $(t, x) \in Q_{\infty}$, there holds

$$
\left|u^{1}(t, x)-u^{2}(t, x)\right| \leq t M\left[\max _{x, \alpha, \beta}\left\|\sigma_{1}-\sigma_{2}\right\|+\max _{x, \alpha, \beta}\left|f_{1}-f_{2}\right|\right]+t\left[\max _{x, \alpha, \beta}\left|\ell_{1}-\ell_{2}\right|+\omega\left(\max _{x, \alpha, \beta}\left\|\sigma_{1}-\sigma_{2}\right\|\right)\right]
$$

where $u^{1}$ and $u^{2}$ are respectively the solution to $(\mathrm{Pi})$ with $i=1$ and $i=2$.

Remark 2.6. Relation (2.14) is fulfilled provided that there exists $A_{i}^{\prime} \subset A$ such that

$$
\sigma_{i}(x, \alpha, \beta)=0 \quad \forall \alpha \in A_{i}^{\prime}, \quad B(0, \nu) \subset \overline{\operatorname{conv}}\left\{f_{i}(x, \alpha, \beta) \mid \alpha \in A_{i}^{\prime}\right\}
$$

for every $x \in \mathbb{R}^{n}, \beta \in B$ (here, $B(0, \nu)$ stands for the ball centered in 0 with radius $\nu$ while conv $F$ is the convex hull of $F \subset \mathbb{R}^{n}$ ).

Proof of Corollary 2.5. A straightforward application of Theorem 2.2 and Remark 2.3 yields the statement provided that the functions $u^{1}$ and $u^{2}$ satisfy condition $(2.2)$ with $\gamma=1$. Let us prove this property by using some arguments of [3], Theorem II.1. Assume that there holds

$$
\left|u^{i}(t, x)-u^{i}(t+h, x)\right| \leq C h, \quad \forall(t, x) \in Q_{\infty}, h>0, i=1,2 .
$$

In this case, relations (2.14) and (2.15) guarantee in viscosity sense

$$
C \geq H\left(x, D u^{i}(\cdot, t), D^{2} u^{i}(\cdot, t)\right) \geq \nu\left|D u^{i}(\cdot, t)\right|-C, \quad \text { in } \mathbb{R}^{n}
$$

for all $t \in[0,+\infty), i=1,2$. In particular, we have: $\left|D u^{i}\right| \leq 2 C \nu^{-1}$, which amounts to (2.2) with $\gamma=1$.

In conclusion, let us prove inequality (2.15). By estimate (2.1), we infer that the functions $u^{i}(t+h, x) \pm C h$ are respectively a super and a subsolution to (Pi). Applying the comparison principle, we accomplish the proof of estimate (2.15).

\section{Estimate FOR THE ERGODIC PROBLEM}

This section is devoted to provide a continuous dependence estimate for the ergodic constant associated to the HJBI operator (1.2) (which we consider the main result of this paper). Let us recall that, in the ergodic problem, we seek a constant $U$ such that the equation

$$
H\left(x, D v, D^{2} v\right)=U \quad \text { in } \mathbb{R}^{n}
$$

admits at least one solution $v$. To this purpose, it is expedient to introduce the approximated equation

$$
\delta w_{\delta}+H\left(x, D w_{\delta}, D^{2} w_{\delta}\right)=0 \quad \text { in } \mathbb{R}^{n} \quad(\delta>0) .
$$

Besides our standing assumptions, throughout this section, the operator $H$ also fulfills

(A4) Periodicity: the functions $\sigma, f$ and $\ell$ are $\mathbb{Z}^{n}$-periodic in $x$. 
We also introduce the following alternative conditions:

(A5) Ellipticity: there exists a positive constant $\nu$ such that:

$$
a(x, \alpha, \beta) \geq \nu I, \quad \forall(x, \alpha, \beta) \in \mathbb{R}^{n} \times A \times B .
$$

(A6) Controllability: for some positive constants $\nu$ and $C_{1}$, there holds

$$
H(x, p, X) \geq \nu|p|-C_{1} \quad \forall(x, p, X) \in \mathbb{R}^{n} \times \mathbb{R}^{n} \times \mathbb{S}^{n} .
$$

For later use, in the following Proposition, we shall collect several known properties of the ergodic problem.

Proposition 3.1. Under assumptions (A1)-(A4) and either (A5) or (A6), the following properties hold:

(i) there exists exactly one constant $U$ such that equation (3.1) admits a bounded continuous (and periodic) solution $v$. Moreover, $v$ is unique up to an additive constant provided that (A5) holds;

(ii) let $u$ be the solution to the Cauchy problem (1.1); then, as $t \rightarrow+\infty, u(t, x) / t$ converges to the ergodic constant $U$ of equation (3.1) uniformly in $x$;

(iii) the approximated equation (3.2) admits exactly one bounded continuous solution $w_{\delta}: \delta\left\|w_{\delta}\right\|_{\infty} \leq \max _{x, \alpha, \beta}|\ell|$. Moreover, as $\delta \rightarrow 0^{+}, \delta w_{\delta}$ and $\left(w_{\delta}-w_{\delta}(0)\right)$ converge respectively to the ergodic constant $U$ and to a solution $v$ of (3.1) with $v(0)=0$;

(iv) there exist a positive constant $\tilde{K}$, depending only on the parameters of our assumptions (that is, independent of $\delta$ ) such that

$$
\left|D w_{\delta}\right| \leq \tilde{K} \quad \text { for a.e. } x \in \mathbb{R}^{n} .
$$

Proof of Proposition 3.1. Under assumption (A6), the detailed proofs are given in [2], Proposition 6.4, (see also [3], Theorem II.1, for operators of Bellman type). Under assumption (A5), the proof of points $(i)$, (ii) and (iii) can be found in [2], Theorem 4.1, (see also [3], Thm. II.2, for Bellman operators); moreover, the proof of point $(i v)$ can be easily obtained adapting to HJBI equations the arguments introduced in [3], Theorem II.2, (see also [2], Thm. 4.1, for a similar result).

Remark 3.2. In fact, by the arguments above, one can prove that there exist $\kappa \in(0,1]$ and $K>0$ (both depending only on the parameters of our assumptions) such that

$$
\left\|w_{\delta}-w_{\delta}(0)\right\|_{C^{1, \kappa}} \leq K\left(1+\max _{x, \alpha, \beta}|\ell|\right)
$$

(see [2], proof of Theorem 4.1, for the special form of the right-hand side) provided that assumption (A5) holds and that there holds

$$
\left|D w_{\delta}\right| \leq \nu^{-1}\left(C_{1}+\max _{x, \alpha, \beta}|\ell|\right)
$$

provided that assumption $(A 6)$ holds.

Assume conditions $(A 1)-(A 4)$ and either $(A 5)$ or $(A 6)$; for $i=1,2$, consider the ergodic problems

$$
\min _{\beta \in B} \max _{\alpha \in A}\left\{-\operatorname{tr}\left(a_{i}(x, \alpha, \beta) D^{2} v^{i}\right)+f_{i}(x, \alpha, \beta) \cdot D v^{i}+\ell_{i}(x, \alpha, \beta)\right\}=U^{i} \quad \text { in } \mathbb{R}^{n} .
$$

Theorem 3.3. Let $U^{i}$ be the unique ergodic constant for problem $(\mathrm{Ei})(i=1,2)$. Then, there holds

$$
\left|U^{1}-U^{2}\right| \leq \tilde{M}\left(\max _{x, \alpha, \beta}\left\|\sigma_{1}-\sigma_{2}\right\|+\max _{x, \alpha, \beta}\left|f_{1}-f_{2}\right|\right)+\omega\left(\max _{x, \alpha, \beta}\left\|\sigma_{1}-\sigma_{2}\right\|\right)+\max _{x, \alpha, \beta}\left|\ell_{1}-\ell_{2}\right|
$$

with $\tilde{M}:=2 \tilde{K}\left(2 C_{\sigma}^{2}+2+C_{f}\right)$, where $\tilde{K}$ is the constant introduced in Proposition 3.1-(iv) while $C_{\sigma}$ and $C_{f}$ are the Lipschitz constants of $\sigma$ and $f$ respectively (see assumption (A3)). 
Remark 3.4. This result is a twofold extension of [2], Theorem 4.5; here, the matrix $\sigma$ depends on the control of both players (hence, the operator $H$ may be not convex in $X$ ) and the running cost $\ell$ is uniformly continuous (instead of being Hölder continuous). In particular, let us stress that, taking advantage of the convexity of $H$ and of the regularity of $\ell$, in [2] an estimate of the $C^{2}$-norm is provided both for the solution of the ergodic problem and for the solution of the approximated equation while, under our assumptions, in general, these functions only belong to $C^{1}$.

This result also extends [2], Corollary 6.9, to the case of different dispersion matrices (i.e., $\left.\sigma_{1} \neq \sigma_{2}\right)$.

Proof of Theorem 3.3. The statement is an immediate consequence of Corollary 2.5 and Proposition 3.1-(ii) provided that assumption $(A 6)$ is fulfilled.

Consider now that condition $(A 5)$ holds. Let $u^{i}$ be the solution to problem (Pi) $(i=1,2)$. By Proposition 3.1(ii), the statement follows from Theorem 2.2 and Remark 2.3 provided that the solutions $u^{1}$ and $u^{2}$ fulfill condition (2.2) with $\gamma=1$. In order to prove this fact, we denote by $v^{i}$ the unique bounded solution to equation (Ei) with $v^{i}(0)=0$ and we introduce the function $w^{i}(t, x):=u^{i}(t, x)+U^{i} t$, which is the unique solution to the Cauchy problem

$$
\begin{cases}\partial_{t} w^{i}+\min _{\beta \in B} \max _{\alpha \in A}\left\{-\operatorname{tr}\left(a_{i} D^{2} w^{i}\right)+f_{i} \cdot D w^{i}+\ell_{i}\right\}=U^{i} & \text { in } Q_{\infty} \\ w^{i}(0, x)=0 & \text { on } \mathbb{R}^{n} .\end{cases}
$$

Let us prove that $w^{i}$ is bounded in $Q_{\infty}$ arguing as in [3], Theorem II.1. For $k:=\left\|v^{i}\right\|_{\infty}$, the functions $v^{i}(x)-$ $U^{i} t \pm k$ are respectively a super- and a subsolution to the Cauchy problem $(\mathrm{Pi})$; hence, the comparison principle ensures

$$
v^{i}(x)-k \leq w^{i}(t, x) \leq v^{i}(x)+k \quad \forall(t, x) \in Q_{\infty},
$$

and, in particular: $\left\|w^{i}\right\|_{\infty} \leq 2 k$. Furthermore, we observe that, as $\delta \rightarrow 0^{+}$, Proposition 3.1-(iii) ensures: $\left|U^{i}\right| \leq \max _{x, \alpha, \beta}\left|\ell_{i}\right|$. In conclusion, by standard regularity theory for parabolic equations (see $[18,35,36]$ ), the functions $w^{i}$ fulfill hypothesis (2.2) with $\gamma=1$ and, consequently, also $u^{i}$ fulfill hypothesis (2.2) with $\gamma=1$.

Proof of Theorem 3.3: alternative version. We shall follow the arguments for the comparison principle (see [17] and also [28] for continuous dependence estimates). For every positive $\eta$, define

$$
\psi(x, y):=w_{\delta}^{1}(x)-w_{\delta}^{2}(y)-\frac{\eta}{2}|x-y|^{2}
$$

where $w_{\delta}^{i}(i=1,2)$ is the unique bounded (and periodic) solution to

$$
\delta w_{\delta}^{i}+\min _{\beta \in B} \max _{\alpha \in A}\left\{-\operatorname{tr}\left(a_{i} D^{2} w_{\delta}^{i}\right)+f_{i} \cdot D w_{\delta}^{i}+\ell_{i}\right\}=0 \quad \text { in } \mathbb{R}^{n} .
$$

(Here, taking advantage of the periodicity of $w_{\delta}^{i}$, the penalization term is simpler than the one in the proof of Theorem 2.2.) Owing to these properties of $w_{\delta}^{i}$, we deduce that there exists a point $\left(x_{0}, y_{0}\right) \in \mathbb{R}^{n} \times \mathbb{R}^{n}$ where the function $\psi$ attains its global maximum.

Let us now claim that, for $\bar{C}:=2 \tilde{K}$ (where $\tilde{K}$ is the constant introduced in Proposition 3.1-(iv)), there holds

$$
\eta\left|x_{0}-y_{0}\right| \leq \bar{C}
$$

Actually, we observe that the inequality $\psi\left(x_{0}, x_{0}\right)+\psi\left(y_{0}, y_{0}\right) \leq 2 \psi\left(x_{0}, y_{0}\right)$ gives

$$
\eta\left|x_{0}-y_{0}\right|^{2} \leq\left[w_{\delta}^{1}\left(x_{0}\right)-w_{\delta}^{1}\left(y_{0}\right)\right]+\left[w_{\delta}^{2}\left(x_{0}\right)-w_{\delta}^{2}\left(y_{0}\right)\right] \leq \bar{C}\left|x_{0}-y_{0}\right|
$$

where the latter inequality is due to Proposition 3.1-(iv) and to the definition of $\bar{C}$; whence, estimate (3.5) easily follows. 
By [17], Theorem 3.2, for every $\nu>0$, there exist matrices $X, Y \in \mathbb{S}^{n}$ such that

$$
\begin{gathered}
\left(\eta\left(x_{0}-y_{0}\right), X\right) \in J^{2,+} w_{\delta}^{1}\left(x_{0}\right), \quad\left(\eta\left(x_{0}-y_{0}\right), Y\right) \in J^{2,-} w_{\delta}^{2}\left(y_{0}\right) \\
\left(\begin{array}{cc}
X & 0 \\
0 & -Y
\end{array}\right) \leq \eta(1+2 \nu \eta)\left(\begin{array}{cc}
I & -I \\
-I & I
\end{array}\right) .
\end{gathered}
$$

Moreover, arguing as in the proof of Theorem 2.2, from last inequality we deduce

$$
\operatorname{tr}\left(a_{1}\left(x_{0}, \alpha, \beta\right) X\right)-\operatorname{tr}\left(a_{2}\left(y_{0}, \alpha, \beta\right) Y\right) \leq \eta(1+2 \nu \eta)\left\|\sigma_{1}\left(x_{0}, \alpha, \beta\right)-\sigma_{2}\left(y_{0}, \alpha, \beta\right)\right\|^{2}
$$

for every $(\alpha, \beta) \in A \times B$. Since $w_{\delta}^{1}$ (respectively, $\left.w_{\delta}^{2}\right)$ is a subsolution (resp., a supersolution) to equation (3.4) with $i=1$ (resp., $i=2$ ), by relations (3.6), we infer

$$
\begin{array}{r}
\delta w_{\delta}^{1}\left(x_{0}\right)+\min _{\beta \in B} \max _{\alpha \in A}\left\{-\operatorname{tr}\left(a_{1}\left(x_{0}, \alpha, \beta\right) X\right)+\eta f_{1}\left(x_{0}, \alpha, \beta\right) \cdot\left(x_{0}-y_{0}\right)+\ell_{1}\left(x_{0}, \alpha, \beta\right)\right\} \leq 0 \\
\delta w_{\delta}^{2}\left(y_{0}\right)+\min _{\beta \in B} \max _{\alpha \in A}\left\{-\operatorname{tr}\left(a_{2}\left(y_{0}, \alpha, \beta\right) Y\right)+\eta f_{2}\left(y_{0}, \alpha, \beta\right) \cdot\left(x_{0}-y_{0}\right)+\ell_{2}\left(y_{0}, \alpha, \beta\right)\right\} \geq 0 .
\end{array}
$$

Taking into account the last three inequalities and arguing as before, we obtain

$$
\begin{aligned}
\delta\left(w_{\delta}^{1}\left(x_{0}\right)-w_{\delta}^{2}\left(y_{0}\right)\right) \leq & \eta(1+2 \nu \eta) \max _{\alpha, \beta}\left\|\sigma_{1}\left(x_{0}, \alpha, \beta\right)-\sigma_{2}\left(y_{0}, \alpha, \beta\right)\right\|^{2}+\eta\left|x_{0}-y_{0}\right| \max _{\alpha, \beta} \mid f_{1}\left(x_{0}, \alpha, \beta\right) \\
& -f_{2}\left(y_{0}, \alpha, \beta\right)\left|+\max _{\alpha, \beta}\right| \ell_{1}\left(x_{0}, \alpha, \beta\right)-\ell_{2}\left(y_{0}, \alpha, \beta\right) \mid .
\end{aligned}
$$

Letting $\nu \rightarrow 0^{+}$, by the regularity of the coefficients (see assumption $(A 3)$ ) and by estimate (3.5), we have

$$
\begin{aligned}
\delta\left(w_{\delta}^{1}\left(x_{0}\right)-w_{\delta}^{2}\left(y_{0}\right)\right) \leq & 2 \eta\left(C_{\sigma}^{2}\left|x_{0}-y_{0}\right|^{2}+\max _{x, \alpha, \beta}\left\|\sigma_{1}-\sigma_{2}\right\|^{2}\right)+\eta C_{f}\left|x_{0}-y_{0}\right|^{2}+\eta\left|x_{0}-y_{0}\right| \max _{x, \alpha, \beta}\left|f_{1}-f_{2}\right| \\
& +\omega\left(\left|x_{0}-y_{0}\right|\right)+\max _{x, \alpha, \beta}\left|\ell_{1}-\ell_{2}\right| \\
\leq & \bar{C}^{2}\left(2 C_{\sigma}^{2}+C_{f}\right) \eta^{-1}+2 \eta \max _{x, \alpha, \beta}\left\|\sigma_{1}-\sigma_{2}\right\|^{2}+\bar{C} \max _{x, \alpha, \beta}\left|f_{1}-f_{2}\right|+\omega\left(\bar{C} \eta^{-1}\right)+\max _{x, \alpha, \beta}\left|\ell_{1}-\ell_{2}\right| .
\end{aligned}
$$

As $\delta \rightarrow 0^{+}$, we infer

$$
U^{1}-U^{2} \leq \bar{C}^{2}\left(2 C_{\sigma}^{2}+C_{f}\right) \eta^{-1}+2 \eta \max _{x, \alpha, \beta}\left\|\sigma_{1}-\sigma_{2}\right\|^{2}+\bar{C} \max _{x, \alpha, \beta}\left|f_{1}-f_{2}\right|+\omega\left(\bar{C} \eta^{-1}\right)+\max _{x, \alpha, \beta}\left|\ell_{1}-\ell_{2}\right| .
$$

Arguing as in the proof of Theorem 2.2 (with $\gamma=1$ ), we ascertain one side of our statement. Reversing the role of $w_{\delta}^{1}$ and $w_{\delta}^{2}$, one can easily obtain the other side; therefore, we shall omit its proof.

\subsection{Singular perturbation problems}

We consider the following singular perturbation problems

$$
\begin{cases}\partial_{t} u^{\varepsilon}+\mathcal{H}\left(x, y, D_{x} u^{\varepsilon}, \frac{D_{y} u^{\varepsilon}}{\varepsilon}, D_{x x}^{2} u^{\varepsilon}, \frac{D_{y y}^{2} u^{\varepsilon}}{\varepsilon}, \frac{D_{x y}^{2} u^{\varepsilon}}{\sqrt{\varepsilon}}\right)=0 & \text { in }(0, T) \times \mathbb{R}^{n} \times \mathbb{R}^{m} \\ u^{\varepsilon}(0, x, y)=h(x) & \text { on } \mathbb{R}^{n} \times \mathbb{R}^{m}\end{cases}
$$

where $u^{\varepsilon}=u^{\varepsilon}(t, x, y)$ is a real function, $\varepsilon \in(0,1)$ and

$$
\mathcal{H}(x, y, p, q, X, Y, Z):=\min _{\beta \in B} \max _{\alpha \in A}\{-\operatorname{tr}(M X)-\operatorname{tr}(N Y)-2 \operatorname{tr}(E Z)+F \cdot q+G \cdot p+L\}
$$

with $\phi=\phi(x, y, \alpha, \beta)$ for every $\phi=M, N, E, F, G, L$. The aim of this section is to study the asymptotic behavior of $u^{\varepsilon}$ as $\varepsilon \rightarrow 0^{+}$. For the wide literature on this matter, we refer the reader to the monographs by Bensoussan [10], 
Dontchev and Zolezzi [20], Kokotović et al. [31], Alvarez and Bardi [2] and references therein. Let us only recall that these problems arise in zero-sum two-persons stochastic differential games (1.3)-(1.4) where the state variable "splits" in the slow one $x$ and in the fast one $y$. For the control system

$$
\begin{array}{ll}
\mathrm{d} x_{s}=G\left(x_{s}, y_{s}, \alpha_{s}, \beta_{s}\right) \mathrm{d} s+\sqrt{2} \Xi\left(x_{s}, y_{s}, \alpha_{s}, \beta_{s}\right) \mathrm{d} W_{s}, & x_{0}=x \\
d y_{s}=\varepsilon^{-1} F\left(x_{s}, y_{s}, \alpha_{s}, \beta_{s}\right) \mathrm{d} s+\sqrt{2 \varepsilon^{-1}} \Sigma\left(x_{s}, y_{s}, \alpha_{s}, \beta_{s}\right) \mathrm{d} W_{s}, & y_{0}=y
\end{array}
$$

and the cost functional

$$
P(t, x, y, \alpha, \beta):=\mathbb{E}_{(x, y)}\left[\int_{0}^{t} L\left(x_{s}, y_{s}, \alpha_{s}, \beta_{s}\right) \mathrm{d} s+h\left(x_{t}\right)\right],
$$

the lower value function $u^{\varepsilon}$ is a viscosity solution to problem (3.7) with $M=\Xi \Xi^{T}, N=\Sigma \Sigma^{T}$ and $E=\Sigma \Xi^{T}$.

Throughout this section, for some positive constants $C$ and $\nu$, we shall assume:

(S1) $A$ and $B$ are two compact metric spaces.

(S2) $M=\Xi \Xi^{T}, N=\Sigma \Sigma^{T}, E=\Sigma \Xi^{T}$. The functions $\Xi, \Sigma, F, G, L$ and $h$ are bounded continuous functions in $\mathbb{R}^{n} \times \mathbb{R}^{m} \times A \times B$ with values respectively in $\mathbb{M}^{n, p}, \mathbb{M}^{m, p}, \mathbb{R}^{m}, \mathbb{R}^{n}, \mathbb{R}$ and $\mathbb{R}$, namely there holds: $\|\phi\|_{\infty} \leq C$ for $\phi=M, N, E, F, G, L$.

All these functions are $\mathbb{Z}^{m}$-periodic in $y$.

(S3) The functions $\Xi, \Sigma, F$ and $G$ (respectively, $L$ and $h$ ) are Lipschitz (resp., uniformly) continuous in $(x, y)$ uniformly in $(\alpha, \beta)$ that is: there exists a positive constant $C_{\phi}$ and a modulus of continuity $\omega_{\psi}$ such that

$$
\begin{aligned}
\left|\phi\left(x_{1}, y_{1}, \alpha, \beta\right)-\phi\left(x_{2}, y_{2}, \alpha, \beta\right)\right| & \leq C_{\phi}\left(\left|x_{1}-x_{2}\right|+\left|y_{1}-y_{2}\right|\right) \\
\left|\psi\left(x_{1}, y_{1}, \alpha, \beta\right)-\psi\left(x_{2}, y_{2}, \alpha, \beta\right)\right| & \leq \omega_{\psi}\left(\left|x_{1}-x_{2}\right|+\left|y_{1}-y_{2}\right|\right)
\end{aligned}
$$

for every $\left(x_{i}, y_{i}\right) \in \mathbb{R}^{n} \times \mathbb{R}^{m}(i=1,2)$ and $(\alpha, \beta) \in A \times B$, with $\phi=\Xi, \Sigma, F, G$ and $\psi=L, h$.

(S4) For every $(x, y, \alpha, \beta) \in \mathbb{R}^{n} \times \mathbb{R}^{m} \times A \times B$, there holds: $M(x, y, \alpha, \beta) \geq \nu I_{n}$.

and either

(S5) For every $(x, y, \alpha, \beta) \in \mathbb{R}^{n} \times \mathbb{R}^{m} \times A \times B$, there holds: $N(x, y, \alpha, \beta) \geq \nu I_{m}$.

or

(S6) For every $(x, y, p, q, X, Y) \in \mathbb{R}^{n} \times \mathbb{R}^{m} \times \mathbb{R}^{n} \times \mathbb{R}^{m} \times \mathbb{S}^{n} \times \mathbb{S}^{m}$, there holds

$$
\mathcal{H}(x, y, p, q, X, Y, 0) \geq \nu|q|-C(1+|p|+\|X\|) .
$$

Remark 3.5. Under assumptions $(S 1)$ and $(S 2)$, condition $(S 6)$ is fulfilled provided that there exists $A^{\prime} \subset A$ such that

$$
\Sigma(x, y, \alpha, \beta)=0 \quad \forall \alpha \in A^{\prime}, \quad B(0, \nu) \subset \overline{\operatorname{conv}}\left\{F(x, y, \alpha, \beta) \mid \alpha \in A^{\prime}\right\}
$$

for every $(x, y, \beta) \in \mathbb{R}^{n} \times \mathbb{R}^{m} \times B$.

As in $[1,2]$, let us introduce the effective Hamiltonian $\bar{H}$ in the following manner: for every $(\bar{x}, \bar{p}, \bar{X}) \in$ $\mathbb{R}^{n} \times \mathbb{R}^{n} \times \mathbb{S}^{n}$ fixed, the value $-\bar{H}(\bar{x}, \bar{p}, \bar{X})$ is the ergodic constant for $\mathcal{H}(\bar{x}, y, \bar{p}, q, \bar{X}, Y, 0)$ with respect to the variable $y$. We refer the reader to Proposition 3.1 for several properties of the ergodic problem; in particular, let us recall that, for $\delta>0$, the problem

$$
\delta w_{\delta}+\mathcal{H}\left(\bar{x}, y, \bar{p}, D_{y} w, \bar{X}, D_{y y}^{2} w, 0\right)=0 \quad \text { in } \mathbb{R}^{m}, \quad w_{\delta}=w_{\delta}(y) \quad \text { periodic }
$$

admits exactly one continuous solution and moreover, as $\delta \rightarrow 0^{+}, \delta w_{\delta}$ uniformly converges to the value $-\bar{H}(\bar{x}, \bar{p}, \bar{X})$. Let us also emphasize (see also [2], Thm. 4.1, and Rem. 3.2) that Proposition 3.1-(iv) can be stated as follows: there exist $K>0$ such that

$$
\left|D w_{\delta}\right| \leq \tilde{K}:=K(1+|\bar{p}|+\|\bar{X}\|) \quad \text { for a.e. } y \in \mathbb{R}^{m},
$$

for every $(\bar{x}, \bar{p}, \bar{X}) \in \mathbb{R}^{n} \times \mathbb{R}^{n} \times \mathbb{S}^{n}$ and $\delta>0$. 
Corollary 3.6. The solution $u^{\varepsilon}$ to problem (3.7) converges locally uniformly in $[0, T) \times \mathbb{R}^{n} \times \mathbb{R}^{m}$ to the unique solution $u=u(t, x)$ to the effective problem

$$
\begin{cases}\partial_{t} u+\bar{H}\left(x, D_{x} u, D_{x x}^{2} u\right)=0 & \text { in }(0, T) \times \mathbb{R}^{n} \\ u(0, x)=h(x) & \text { on } \mathbb{R}^{n} .\end{cases}
$$

Remark 3.7. This result is a twofold extension of [2], Theorem 4.5: here, the running cost $L$ is only uniformly continuous (namely, its Hölder continuity is not required) and the matrix $\Sigma$ depends on the controls of both players (thus, the Hamiltonian in (3.8) may be not convex in $Y$ ).

This result also extends [2], Corollary 6.9 , to the case where the matrix $\Sigma$ depends on $x$ as well.

Proof of Corollary 3.6. We shall argue using several results established by Alvarez and Bardi [1,2]. Invoking [2], Theorem 2.9, it suffices to prove that the comparison principle applies to the effective problem (3.10). To this end, by virtue of the results by Ishii and Lions [25], it suffices to establish the following two properties: $(i) \bar{H}$ is uniformly elliptic, (ii) for some constant $\bar{K}$ and for some modulus of continuity $\bar{\omega}$, there holds

$$
\begin{aligned}
\left|\bar{H}\left(x_{1}, p_{1}, X_{1}\right)-\bar{H}\left(x_{2}, p_{2}, X_{2}\right)\right| \leq & C\left\|X_{1}-X_{2}\right\|+C\left|p_{1}-p_{2}\right|+\bar{\omega}\left(\left|x_{1}-x_{2}\right|\right) \\
& +\bar{K}\left|x_{1}-x_{2}\right|\left(1+\left|p_{1}\right| \vee\left|p_{2}\right|+\left\|X_{1}\right\| \vee\left\|X_{2}\right\|\right)
\end{aligned}
$$

for every $\left(x_{i}, p_{i}, X_{i}\right) \in \mathbb{R}^{n} \times \mathbb{R}^{n} \times \mathbb{S}^{n}(i=1,2)$. We observe that the uniform ellipticity is well known so we shall omit its proof and we refer the reader to [2], Theorem 4.4, for the detailed proof. In order to prove (3.11), let us recall that, for $i=1,2$, the value $-\bar{H}\left(x_{i}, p_{i}, X_{i}\right)$ is the ergodic constant for the problem

$$
\begin{aligned}
\min _{\beta \in B} \max _{\alpha \in A}\left\{-\operatorname{tr}\left(N\left(x_{i}, y, \alpha, \beta\right) D_{y y}^{2} w^{i}\right)+D_{y} w^{i} \cdot F\left(x_{i}, y, \alpha, \beta\right)-\operatorname{tr}\left(M\left(x_{i}, y, \alpha, \beta\right) X_{i}\right)\right. \\
\left.+p_{i} \cdot G\left(x_{i}, y, \alpha, \beta\right)+L\left(x_{i}, y, \alpha, \beta\right)\right\}=-\bar{H}\left(x_{i}, p_{i}, X_{i}\right) .
\end{aligned}
$$

Applying Theorem 3.3 with the variable $x$ replaced by $y$ and

$$
\begin{aligned}
\sigma_{i}(\cdot, \alpha, \beta) & =\Sigma\left(x_{i}, \cdot, \alpha, \beta\right), \quad f_{i}(\cdot, \alpha, \beta)=F\left(x_{i}, \cdot, \alpha, \beta\right) \\
\ell_{i}(\cdot, \alpha, \beta) & =-\operatorname{tr}\left(M\left(x_{i}, \cdot, \alpha, \beta\right) X_{i}\right)+p_{i} \cdot G\left(x_{i}, \cdot, \alpha, \beta\right)+L\left(x_{i}, \cdot, \alpha, \beta\right) \\
\omega(r) & =\left[C_{M}\left(\left\|X_{1}\right\| \vee\left\|X_{2}\right\|\right)+C_{G}\left(\left|p_{1}\right| \vee\left|p_{2}\right|\right)\right] r+\omega_{L}(r)
\end{aligned}
$$

for some constant $\tilde{M}$ (see below for its dependence on $\left(x_{i}, p_{i}, X_{i}\right)$ ), we infer

$$
\begin{aligned}
\left|\bar{H}\left(x_{1}, p_{1}, X_{1}\right)-\bar{H}\left(x_{2}, p_{2}, X_{2}\right)\right| \leq & \tilde{M}\left(\max _{y, \alpha, \beta}\left\|\Sigma\left(x_{1}, y, \alpha, \beta\right)-\Sigma\left(x_{2}, y, \alpha, \beta\right)\right\|+\max _{y, \alpha, \beta}\left|F\left(x_{1}, y, \alpha, \beta\right)-F\left(x_{2}, y, \alpha, \beta\right)\right|\right) \\
& +\left[C_{M}\left(\left\|X_{1}\right\| \vee\left\|X_{2}\right\|\right)+C_{G}\left(\left|p_{1}\right| \vee\left|p_{2}\right|\right)\right] \max _{y, \alpha, \beta}\left\|\Sigma\left(x_{1}, y, \alpha, \beta\right)-\Sigma\left(x_{2}, y, \alpha, \beta\right)\right\| \\
& +\omega_{L}\left(\max _{y, \alpha, \beta}\left\|\Sigma\left(x_{1}, y, \alpha, \beta\right)-\Sigma\left(x_{2}, y, \alpha, \beta\right)\right\|\right)+\max _{y, \alpha, \beta} \mid \operatorname{tr}\left[M\left(x_{1}, y, \alpha, \beta\right) X_{1}\right. \\
& \left.-M\left(x_{2}, y, \alpha, \beta\right) X_{2}\right]\left|+\max _{y, \alpha, \beta}\right| p_{1} \cdot G\left(x_{1}, y, \alpha, \beta\right)-p_{2} \cdot G\left(x_{2}, y, \alpha, \beta\right) \mid \\
& +\max _{y, \alpha, \beta}\left|L\left(x_{1}, y, \alpha, \beta\right)-L\left(x_{2}, y, \alpha, \beta\right)\right| .
\end{aligned}
$$

Taking into account the regularity of the coefficients (see assumption $(S 3)$ ), we deduce

$$
\begin{aligned}
\left|\bar{H}\left(x_{1}, p_{1}, X_{1}\right)-\bar{H}\left(x_{2}, p_{2}, X_{2}\right)\right| \leq & C\left\|X_{1}-X_{2}\right\|+C\left|p_{1}-p_{2}\right|+\omega_{L}\left(C_{\Sigma}\left|x_{1}-x_{2}\right|\right)+\omega_{L}\left(\left|x_{1}-x_{2}\right|\right) \\
& +\left|x_{1}-x_{2}\right| \tilde{M}\left(C_{\Sigma}+C_{F}\right)+\left|x_{1}-x_{2}\right|\left[C_{M}\left(\left\|X_{1}\right\| \vee\left\|X_{2}\right\|\right)+C_{G}\left(\left|p_{1}\right| \vee\left|p_{2}\right|\right)\right] C_{\Sigma} \\
& +C_{M}\left|x_{1}-x_{2}\right|\left(\left\|X_{1}\right\| \wedge\left\|X_{2}\right\|\right)+C_{G}\left|x_{1}-x_{2}\right|\left(\left|p_{1}\right| \wedge\left|p_{2}\right|\right) .
\end{aligned}
$$


Since there holds $\max _{x, \alpha, \beta, i}\left|\ell_{i}\right| \leq C\left(1+\left|p_{1}\right| \vee\left|p_{2}\right|+\left\|X_{1}\right\| \vee\left\|X_{2}\right\|\right)$, by our definition of $\tilde{M}$ (see Thm. 3.3) and estimate (3.9), we have

$$
\tilde{M} \leq 2 K(C+1)\left(2 C_{\Sigma}^{2}+2+C_{F}\right)\left(1+\left|p_{1}\right| \vee\left|p_{2}\right|+\left\|X_{1}\right\| \vee\left\|X_{2}\right\|\right) .
$$

Hence, the previous inequality becomes

$$
\begin{aligned}
\left|\bar{H}\left(x_{1}, p_{1}, X_{1}\right)-\bar{H}\left(x_{2}, p_{2}, X_{2}\right)\right| \leq & C\left\|X_{1}-X_{2}\right\|+C\left|p_{1}-p_{2}\right|+\omega_{L}\left(C_{\Sigma}\left|x_{1}-x_{2}\right|\right) \\
& +\omega_{L}\left(\left|x_{1}-x_{2}\right|\right)+\bar{K}\left|x_{1}-x_{2}\right|\left(1+\left|p_{1}\right| \vee\left|p_{2}\right|+\left\|X_{1}\right\| \vee\left\|X_{2}\right\|\right)
\end{aligned}
$$

for some constant $\bar{K}$ independent of $\left(x_{i}, p_{i}, X_{i}\right)$. Finally, setting $\bar{\omega}(r):=\omega_{L}\left(C_{\Sigma} r\right)+\omega_{L}(r)$, our claim (3.11) is completely proved.

\section{REFERENCES}

[1] O. Alvarez and M. Bardi, Singular perturbations of nonlinear degenerate parabolic PDEs: a general convergence result. Arch. Rational Mech. Anal. 170 (2003) 17-61.

[2] O. Alvarez and M. Bardi, Ergodicity, stabilization, and singular perturbations for Bellman-Isaacs equation. Mem. Amer. Math. Soc. 204 (2010).

[3] M. Arisawa and P.L. Lions, On ergodic stochastic control. Comm. Partial Differential Equations 23 (1998) $2187-2217$.

[4] V.I. Arnold and A. Avez, Problèmes ergodiques de la mècanique classique. Gauthiers-Villars, Paris (1967).

[5] G. Barles and F. Da Lio, On the boundary ergodic problem for fully nonlinear equations in bounded domains with general nonlinear Neumann boundary conditions. Ann. Inst. Henri Poincaré, Anal. Non Linéaire 22 (2005) 521-541.

[6] G. Barles, F. Da Lio, P.L. Lions and P.E. Souganidis, Ergodic problems and periodic homogenization for fully nonlinear equations in half-space type domains with Neumann boundary conditions. Indiana Univ. Math. J. 57 (2008) $2355-2375$.

[7] G. Barles and E.R. Jakobsen, Error bounds for monotone approximation schemes for parabolic Hamilton-Jacobi-Bellman equations. Math. Comp. 76 (2007) 1861-1893.

[8] G. Barles, O. Ley and H. Mitake, Short time uniqueness results for solutions of nonlocal and non-monotone geometric equations. arXiv: 1005.5597.

[9] G. Barles and P.E. Souganidis, Space-time periodic solutions and long-time behavior of solutions to quasi-linear parabolic equations. SIAM J. Math. Anal. 32 (2001) 1311-1326.

[10] A. Bensoussan, Perturbation Methods in Optimal Control. Wiley/Gauthiers-Villars, Chichester (1988).

[11] A. Bensoussan, J.L. Lions and G. Papanicolaou, Asymptotic Analysis for periodic Structures. North-Holland, Amsterdam (1978).

[12] I.H. Biswas, E.R. Jakobsen and K.H. Karlsen, Viscosity solutions for a system of integro-PDEs and connections to optimal switching and control of jump-diffusion processes. Appl. Math. Optim. 62 (2010) 47-80.

[13] M. Bourgoing, $C^{1, \beta}$ regularity of viscosity solutions via a continuous-dependence result. Adv. Differential Equations 9 (2004) $447-480$.

[14] L. Caffarelli, P. Souganidis and L. Wang, Homogenization of fully nonlinear, uniformly elliptic and parabolic partial differential equations in stationary ergodic media. Comm. Pure Appl. Math. 58 (2005) 319-361.

[15] B. Cockburn, G. Gripenberg and S.-O. Londen, Continuous dependence on the nonlinearity of viscosity solutions of parabolic equations. J. Differential Equations 170 (2001) 180-187.

[16] I.P. Cornfeld, S.V. Fomin and Y.G. Sinai, Ergodic theory. Springer-Verlag, Berlin (1982).

[17] M.G. Crandall, H. Ishii and P.-L. Lions, User's guide to viscosity solutions of second order partial differential equations. Bull. Amer. Math. Soc. (N.S.) 27 (1992) 1-67.

[18] M.G. Crandall, M. Kocan and A. Świech, $L^{p}$-theory for fully nonlinear uniformly parabolic equations. Comm. Partial Differential Equations 25 (2000) 1997-2053.

[19] H. Dong and N.V. Krylov, The rate of convergence of finite-difference approximations for parabolic Bellman equations with Lipschitz coefficients in cylindrical domains. Appl. Math. Optim. 56 (2007) 37-66.

[20] A. Dontchev and T. Zolezzi, Well-posed Optimization Problems, Lecture Notes in Math. 1543. Berlin (1993).

[21] L. Evans, Periodic homogenisation of certain fully nonlinear partial differential equations. Proc. Roy. Soc. Edinb. Sect. A 120 (1992) 245-265.

[22] W.H. Fleming and P.E. Souganidis, On the existence of value functions of two-players zero-sum stochastic differential games. Indiana Univ. Math. J. 38 (1989) 293-314.

[23] G. Gripenberg, Estimates for viscosity solutions of parabolic equations with Dirichlet boundary conditions. Proc. Am. Math. Soc. 130 (2002) 3651-3660.

[24] H. Ishii, On uniqueness and existence of viscosity solutions of fully nonlinear second-order elliptic PDE's. Comm. Pure Appl. Math. 42 (1989) 15-45. 
[25] H. Ishii and P.L. Lions, Viscosity solutions of fully nonlinear second-order elliptic partial differential equations. J. Differential Equations 83 (1990) 26-78.

[26] E.R. Jakobsen and C.A. Georgelin, Continuous dependence results for non-linear Neumann type boundary value problems. $J$. Differential Equations 245 (2008) 2368-2396.

[27] E.R. Jakobsen and K.H. Karlsen, Continuous dependence estimates for viscosity solutions of fully nonlinear degenerate parabolic equations. J. Differential Equations 183 (2002) 497-525.

[28] E.R. Jakobsen and K.H. Karlsen, Continuous dependence estimates for viscosity solutions of fully nonlinear degenerate elliptic equations. Electron. J. Differential Equations 39 (2002) 1-10.

[29] E.R. Jakobsen and K.H. Karlsen, Continuous dependence estimates for viscosity solutions of integro-PDEs. J. Differential Equations 212 (2005) 278-318.

[30] V.V. Jikov, S.M. Kozlov and O.A. Oleinik, Homogenization of Differential Operators and Integral Functionals. Springer, Berlin (1994).

[31] P.V. Kokotović, H.K. Khalil and J. O'Reilly, Singular perturbation methods in control: analysis and design. Academic Press, London (1986).

[32] P.L. Lions and P. Souganidis, Homogenization of degenerate second-order PDE in periodic and almost periodic environments and applications. Ann. Inst. Henti Poincaré, Anal. Non Linéaire 22 (2005) 667-677.

[33] B. Simon, Functional integration and quantum physics. Academic Press, New York (1979).

[34] P.E. Souganidis, Existence of viscosity solutions of Hamilton-Jacobi equations. J. Differential Equations 56 (1985) 345-390.

[35] L. Wang, On the regularity theory of fully nonlinear parabolic equations: I. Comm. Pure Appl. Math. 45 (1992) $27-76$.

[36] L. Wang, On the regularity theory of fully nonlinear parabolic equations: II. Comm. Pure Appl. Math. 45 (1992) 141-178. 